\title{
Cardiovascular Disease in Ageing: An Overview on Thoracic Aortic Aneurysm as an Emerging Inflammatory Disease
}

\author{
Calogera Pisano, ${ }^{1}$ Carmela Rita Balistreri, ${ }^{2}$ Alessandro Ricasoli, ${ }^{1}$ and Giovanni Ruvolo ${ }^{3}$ \\ ${ }^{1}$ Cardiac Surgery Unit, "P. Giaccone" University Hospital, Palermo, Italy \\ ${ }^{2}$ Department of Pathobiology and Medical and Forensic Biotechnologies, University of Palermo, Palermo, Italy \\ ${ }^{3}$ Cardiac Surgery Unit, Tor Vergata University Hospital, Rome, Italy \\ Correspondence should be addressed to Calogera Pisano; bacalipi@libero.it
}

Received 29 June 2017; Revised 16 September 2017; Accepted 28 September 2017; Published 24 October 2017

Academic Editor: Giuseppe Valacchi

Copyright ( 92017 Calogera Pisano et al. This is an open access article distributed under the Creative Commons Attribution License, which permits unrestricted use, distribution, and reproduction in any medium, provided the original work is properly cited.

\begin{abstract}
Medial degeneration associated with thoracic aortic aneurysm and acute aortic dissection was originally described by Erdheim as a noninflammatory lesion related to the loss of smooth muscle cells and elastic fibre fragmentation in the media. Recent evidences propose the strong role of a chronic immune/inflammatory process in aneurysm evocation and progression. The coexistence of inflammatory cells with markers of apoptotic vascular cell death in the media of ascending aorta with aneurysms and type A dissections raises the possibility that activated $\mathrm{T}$ cells and macrophages may contribute to the elimination of smooth muscle cells and degradation of the matrix. On the other hand, several inflammatory pathways (including TGF- $\beta$, TLR- 4 interferon- $\gamma$, chemokines, and interferon $-\gamma$ ) seem to be involved in the medial degeneration related to aged and dilated aorta. This is an overview on thoracic aortic aneurysm as an emerging inflammatory disease.
\end{abstract}

\section{Introduction}

The average lifespan of the human population is increasing worldwide, mostly because of declining fertility and increasing longevity. It has been predicted that, in 2035, nearly one in four individuals will be 65 years or older [1]. As supported by the growing evidence, age constitutes the crucial risk factor for the development of cardiovascular diseases (CVDs). Accordingly, their prevalence dramatically increases with increasing age [2]. In such association, emerging data that are underlining the crucial involvement of pathophysiological pathways in cardiovascular ageing, dysfunction, and CVD onset are also confirmed by literature [1-6]. Insights and advances in this field are encouraged. This might permit of identifying pathways closely implicated in cardiovascular pathophysiology and useful for improving management, outcomes, and prevention of human CVDs. Today, it is possible to distinguish diverse levels of ageing in cardiovascular system. For instance, in 2017, Steenman and Lande summarized four levels of ageing of heart: functional, structural, cellular, and molecular [7]. Functional changes in the aged heart include systolic and diastolic dysfunction and alterations of the cardiac electrical system [8-11]. Structural changes determine an increase in thickness of heart $[12,13]$ and vessel's wall [14]. Cellular changes include fibrosis [15], medial necrosis and apoptosis [16], and amyloid deposition [17]. Furthermore, the involvement of many molecular pathways implicated in cardiac aging is emerging, even if their role is well-discovered and understood. In regard to vascular system, the main vessel, that is the aorta, as the entire cardiovascular system or any other organ, tissue, and system of human body, shows several modifications with advancing age [18] in all its sections. In particular, in thoracic aorta section, object of our dissertation, diverse age-related changes occur and are responsible for onset of thoracic aorta dysfunction and pathological entities, including medial degeneration (MD) and remodelling. These last assume the role of the catalytic and accelerator drivers in the onset and progression of pathological complications, including the first and more common, the sporadic thoracic ascending aneurysm (S-TAA) [19]. This leads to evidence that S-TAA risk increases when one reaches 65 years old. However, numerous gaps related to the effective 


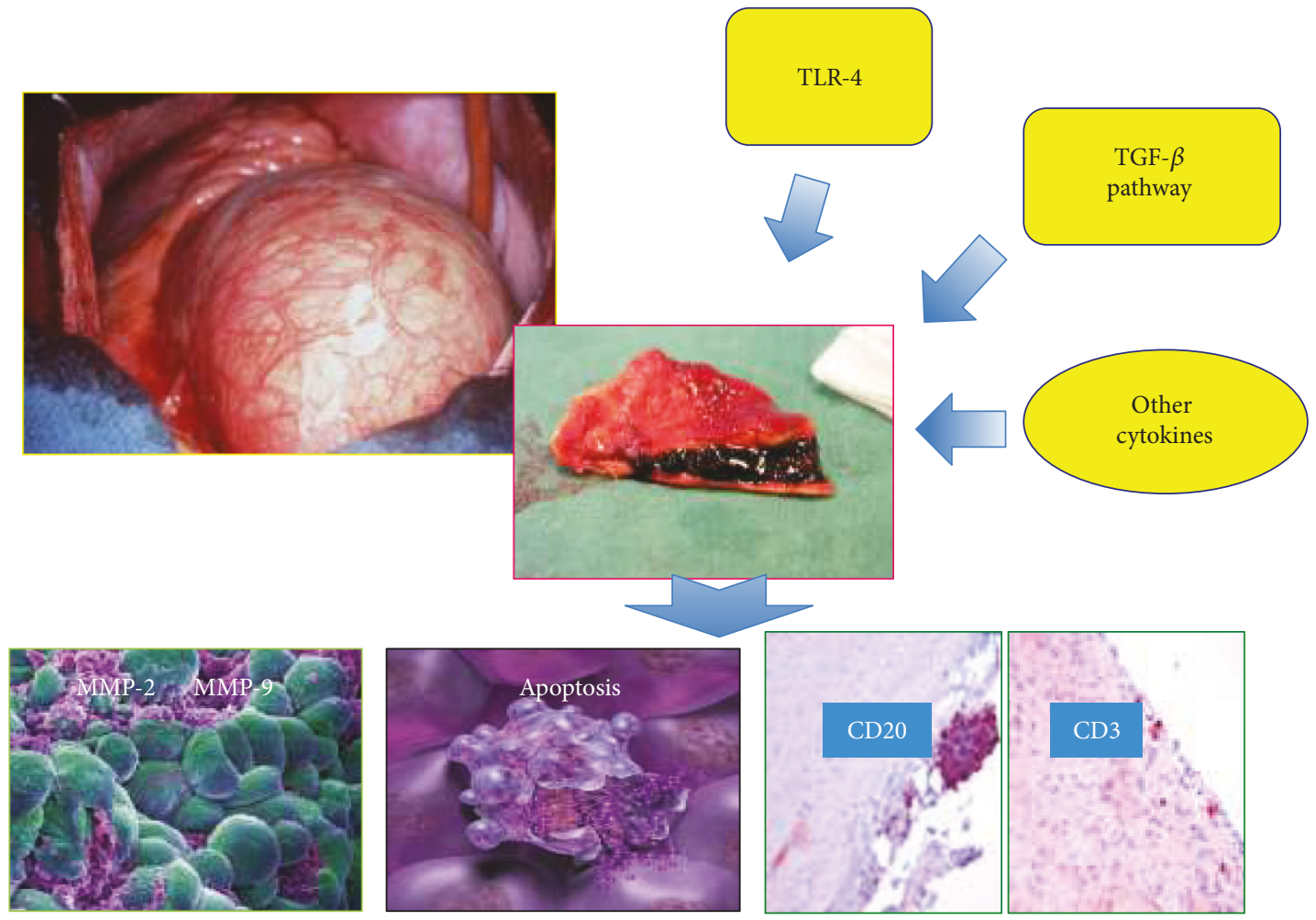

FIGURE 1: Thoracic aortic aneurysm as an emerging inflammatory disease.

cellular and molecular mechanisms remain to be resolved. In this context, the detection of specific histopathological MD phenotypes in old people, associated with a major risk for S-TAA onset and its complications, might be of particular help. We have recently identified a particular MD phenotype significantly associated with the onset of S-TAA in aged individuals, even if based on a small sample size that we have suggested as a potential biomarker. Emerging literature data are also evidencing the key involvement of an upregulation of metalloproteinases (MMPs) and apoptosis, sterile chronic inflammation, and genetic factors in the complex pathophysiology of S-TAA [19]. They are in agreement with recent data, proposing that the sterile inflammation and the related mediators, including cytokines, MMPs, and death mediator, are the shared pathological mechanism for the onset of type A aortic dissection (TAD) [19, 20]. Based on these observations, we reported an overview on emerging literature data about the implication of inflammation in S-TAA pathophysiology Figure 1.

\section{Inflammatory Features of Thoracic Ascending Aneurysm}

Well-recognized variations in the onset and progression characterize TAA and abdominal aortic aneurysm (AAA). However, inflammation is emerging as a common mechanism in the complex, yet different, pathophysiology of the two types of aorta aneurysms [19]. Likewise to AAA, histological studies demonstrate the presence of high levels of inflammatory cells in the adventitia and media of thoracic aortic wall. In particular, it has been demonstrated that macrophages and T lymphocytes are prevalently observed in the thoracic aorta samples of patients affected by sporadic TAA [20-23]. Furthermore, a number of other studies documented the evidences of an increased apoptosis of smooth muscle cells (SMCs) in MD [24, 25]. Accordingly, immunohistochemical studies demonstrated that $\mathrm{MD}$ is associated with levels of p53, a Bax upregulation, and apoptosis of SMC in the aorta of patients with TAD [26]. High levels of Fas (cell surface death receptor) have been diffusely assessed into the aortic media and adventitia. T lymphocytes seem to be the cells responsible for FasL production, in contrast, Fas was found diffusely throughout the media and adventitia. These results suggest apoptosis of primarily SMCs through the Fas-FasL pathway in aneurysmal tissue [22]. The T lymphocyte and macrophage infiltration and TUNEL staining were more pronounced in the media of the dissected aortas than in the aneurysmal aortas. The dissection of blood into the wall of the aorta is a traumatic tissue injury, and it is, therefore, not surprising that inflammatory and apoptotic markers would be increased in the damaged tissue [22]. Although both inflammatory and TUNEL-positive cells were observed along the margin of the dissections, the inflammatory cells and apoptotic markers were present throughout the media as well. It is possible that the degree of inflammation and apoptosis in the media may be increased in an aorta before dissection. These events could potentially lead to increase the rates of loss of SMCs and the destruction of the tissue, setting the stage for the dissection to occur, despite the aortic dimensions. Accordingly, unlike the current recommendations [27], recent studies demonstrated that the diameter is not the only parameter that needs to be 
considered in order to prevent dissection [24, 25, 28]. Some individuals have dissection when the aorta is minimally enlarged, and it is necessary to determine whether this premature dissection is related to the degree of inflammation and apoptosis in the aortic media. In fact, it seems that patients who developed premature dissection carried a particular genetic risk profile characterized by single-nucleotide polymorphisms (SNPs) in inflammatory genes $(-786 \mathrm{~T} / \mathrm{C}$ eNOs, D/IACE, -1562 C/T MMP-9, and -735C/T MMP-2) that causes an important inflammatory reaction, severe tissue injury, and vascular matrix remodelling [29-33].

\section{Role of Cytokines in TAA Pathogenesis}

Several cytokines seem to be involved in TAA formation and complications (rupture and dissection). Among these, transforming growth factor beta (TGF- $\beta$ ) is recognized to have a crucial role in S-TAA pathogenesis, causing extracellular matrix (ECM) degeneration through the production of plasminogen activators and the release of metalloproteinases-2 (MMP-2) and metalloproteinases-9 (MMP-9) [34]. TGF- $\beta$ family consists of TGF- $\beta 1$, TGF- $\beta 2$, and TGF- $\beta 3$ members, which are pleiotropic-secreted cytokines having a broad spectrum of biologic functions. Among these, the TGF- $\beta 1$ has numerous cellular functions, including cell growth, cell proliferation, cell differentiation, and apoptosis. In humans, TGF- $\beta 1$ can stimulate or inhibit cell growth in diverse cellular and tissue targets. In addition, TGF- $\beta 1$ can modulate cell differentiation and proliferation through both canonical and noncanonical pathways [35]. The canonical pathway involves the activation of the TGF- $\beta$ receptor 1 , the phosphorylation of regulatory small mother against decapentaplegic proteins (SMADs 2 and 3), the recruitment of SMAD4 to form a SMAD2/3-SMAD4 complex, the importation into the nucleolus, and activation of gene transcription [36]. Normally, there is an autoregulatory feedback loop on TGF- $\beta$ signalling provided by the canonical pathway [37]. However, it is believed that the loss of functional mutations in genes encoding TGF- $\beta$ pathway leads to an upregulation of TGF$\beta$ signalling, especially through the noncanonical pathways due to loss of this feedback inhibition. The resulting upregulation in TGF- $\beta$ is considered to be a crucial condition strongly associated with the formation of TAA. Based on these observations, we recently assessed the role of five genetic variants of TGF- $\beta$ pathways (TGF- $\beta 1$ and 2 isoforms and $\mathrm{R} 1$ and $\mathrm{R} 2$ receptors) in sporadic TAA. The most relevant finding of this study has allowed us to propose that rs900 TGF- $\beta 2$ SNP is associated with sporadic TAA in women [34]. On the other hand, other reports [38-40] have evidenced a direct or an indirect central role to TGF- $\beta 2$ and its genetic variants in the pathogenesis of both syndromic and familial TAAs. In addition, it has been shown that mutations in the TGFBRII genes deregulate the TGF- $\beta 2$ signalling pathway involved in TAA pathogenesis. TGF- $\beta 2$ gene mutations have been found in familial TAAs and dissections associated with mild systemic features of Marfan syndrome and Loeys-Dietz syndrome and in TAA and dissection associated with mitral valve disease [41]. However, the exact role of TGF- $\beta 2$ in TAA pathogenesis is not clear. In particular, both the genetically determined loss of function and the "paradoxical" augment in the downstream TGF- $\beta$ signalling pathway might be important for TAA development [42]. Furthermore, vascular remodelling, characterizing thoracic aneurysm, seems prevalently to be the result not only of TGF- $\beta$ pathways but also of upregulation of multiple cytokines, including interleukin-10 (IL-10) [43], an antiinflammatory cytokine able to modulate the activity of TGF- $\beta$ pathway, interleukin-6 (IL-6), interleukin-8 (IL-8), tumour necrosis factor- (TNF-) $\alpha$, and monocyte chemoattractant protein-1 [44-46]. On the other hand, interesting studies revealed that circulating levels of interferon- $\gamma$, interferon- $\gamma$-induced chemokines, interferon-inducible T-cell alpha chemoattractant, interferon-inducible protein-10, and monokine induced by interferon gamma are all increased in patients affected by TAAs, even if the levels of these chemokines do not correlate with the size of the aneurysms [47]. Conversely, IL-4 levels have been reported to be reduced in TAA patients [48].

\section{Other Inflammatory Biomarkers}

Systemic levels of inflammatory mediators have been assessed in both acute aortic syndromes (AAD) and TAAs. C-reactive protein (CRP) levels have been observed to increase during AADs [49]. However, it is not clear whether the systemic CRP level increase starts before the onset of these complications or if it is an effect. Regarding this aspect, some studies have shown that a substantial portion of patients with TAA shows normal CRP levels on hospital admission [50]. In contrast, whereas the recent evidence suggests that CRP is increased even in the very early hours after the onset of symptoms [45], this, together with the difficulty in differentiating acute aortic syndromes from other acute cardiovascular problems, such as pulmonary embolism and myocardial infarction, has limited the clinical application of this biomarker for diagnostic purposes. However, the presence of an inflammatory state in both acute aortic syndromes and, to a lesser degree, in chronic TAAs and chronic ADs appears clear. This is evidenced by the increases in both CRP and white blood cell counts [51]. In addition, CRP can be useful for predicting long-term results of acute aortic syndromes. Accordingly, in fact, previous studies have shown that growing CRP levels on admission are associated with a poor prognosis in this setting and that this marker strongly correlates with adverse long-term events at follow-up in Chinese people in both type A (together with white blood cell count) [52] and type B AADs; in this latter case, the most powerful predictor is the CRP peak level after the acute event [53]. Another crucial biomarker is the plasma D-dimer (DD), which is a product of plasmin fibrinolysis of cross-linked fibrin, representing an indirect demonstration of coagulation activation leading to increased fibrin turnover. DD is commonly used for the diagnosis or the exclusion of deep venous thrombosis and pulmonary embolism. However, it is the most studied and likely the most reliable biochemical diagnostic tool in AADs. As an AAD biomarker, DD is quite nonspecific since it can rise in a number of disease states, such as malignancies, recent surgery, disseminated intravascular 
coagulation, deep venous thrombosis, or recent trauma. Nevertheless, several prospective and retrospective studies $[54,55]$ have shown that, with a threshold value of $0.4-$ $0.5 \mu \mathrm{g} / \mathrm{ml}$, AADs can be diagnosed with a high sensitivity, approximately $94-100 \%$, but with a much lower specificity (35-75\%). Interestingly, data from the IRAD-Bio registry suggest that DD has good sensitivity, even in the case of early $(<6 \mathrm{~h})$ presentation [56], although some evidences suggest that testing patients with this biomarker may result in missing the diagnosis of $\mathrm{AAD}$ in up to $18 \%$ of patients [57]. Another major issue about DD is that the assessment of this marker can give a negative result either in the case of a thrombosed false lumen or in the case of an intramural haematoma [58-60]. This further feature limits the chances of using DD, being a solid watershed test for acute aortic syndromes. However, DD may become a more powerful biomarker for monitoring false lumen evolution over time, as hypothesized by some studies [61, 62]. These last evidenced a possible relationship between DD levels and presence of blood flow in the false lumen. Furthermore, a recent meta-analysis [63] of observational studies has suggested some new point on the role of $\mathrm{DD}$ in the diagnosis of $\mathrm{AD}$, showing that the best DD performing the cut-off level is $0.5 \mu \mathrm{g} / \mathrm{ml}$, with a sensitivity of $97 \%$ and a negative predictive value of $96 \%$. On the basis of the results of this meta-analysis, a level of $0.5 \mu \mathrm{g} / \mathrm{ml}$ might be proposed as a rapid and useful screening tool to exclude AADs. This could be of help for the physician in deciding whether to order additional imaging studies to confirm or refuse the diagnosis of $\mathrm{AD}$.

\section{Tole of Toll-Like Receptor-4 in Thoracic Aneurysm Pathogenesis}

Recent evidences underlie the important role of the Toll-like receptor-4 (TLR-4) as key inflammatory promoter for sporadic TAA $[64,65]$. It is a member of Toll-like receptor (TLRs) family. TLRs are the most extensively studied innate receptors, and their roles in innate and adaptive immunity are well-documented [66, 67]. The TLR-4 originally was described as part of the first-line defence against Gramnegative bacteria, it is expressed on leukocytes and a large array of tissue and cell types, such as aortic wall cells, particularly endothelial cells (ECs) and vascular smooth muscle cells (VSMCs), and it responds to particular damage-related products activating a particular inflammatory reaction. In the specific case of sporadic TAA, TLR-4 evocates and modulates the expression and activation of endothelium dysfunction and remodelling aorta pathways. In particular, we suggested a model [68], in which the overstate activation of TLR-4 induced by high levels of danger-associated molecular patterns (DAMPs) determines a typical phenotypic switching of EC and VSMC cells, with the involvement of other pathways, such as stress and stretch pathways. This implies the activation of nuclear factor $-\kappa \mathrm{B}(\mathrm{NF}-\kappa \mathrm{B})$ transcription factor and the production and release of the so-called arterialassociated senescence secretor phenotype (AASSP) characterized by numerous inflammatory mediators, mitotic and trophic factors, proteoglycans and MMPs such as MMP-2 and MMP-9, and vasoactive molecules [31]. In addition, this induces the reduction of nitric oxide (NO) as well. This complex scenario results in modifications of vascular tone and permeability and degradation of components of extracellular matrix (ECM) and elastic fragmentation. Vascular remodelling and $\mathrm{MD}$ are, hence, evocated, which can evolve in aneurysm, dissection, and rupture of the aorta wall.

\section{Evidences about the Role of Inflammation in TAA Complications}

There are several experimental evidences that support inflammatory cells and cytokines as the cause of TAA complications. In a recent study, Tieu et al. [69] showed that subcutaneous infusion of angiotensin II, a vasopressor known to promote vascular inflammation, induced aortic production of the proinflammatory cytokine IL- 6 and the monocyte chemoattractant MCP-1 into older C57BL/6J mice. The production of these factors occurred predominantly in the tunica adventitia, along with macrophage recruitment, adventitial expansion, and development of thoracic and suprarenal aortic dissections. In contrast, a reduced dissection incidence was observed after angiotensin II infusion into mice lacking either IL-6 or the MCP-1 receptor CCR2. Further analysis revealed that angiotensin II induced CCR2 + CD14hiCD11bhiF4/80 - macrophage accumulation selectively in aortic dissections and not in aortas from Il6-/- mice. Adoptive transfer of $\mathrm{Ccr} 2+/+$ monocytes into $\mathrm{Ccr} 2-/-$ mice resulted in selective monocyte uptake into the ascending and suprarenal aorta in regions of enhanced ROS stress, with restoration of IL- 6 secretion and increased incidence of dissection. In vitro, culture of monocytes and aortic adventitial fibroblasts produced MCP-1- and IL-6-enriched conditioned medium that promoted differentiation of monocytes into macrophages, induced CD14 and CD11b upregulation, and induced MCP-1 and MMP-9 expression. These results suggest that leukocyte-fibroblast interactions in the aortic adventitia potentiate IL- 6 production, inducing local monocyte recruitment and activation, thereby promoting MCP-1 secretion, vascular inflammation, ECM remodelling, and aortic destabilization. On the other hand, Kurobe et al. [70] demonstrated that combined treatment with angiotensin II and $\beta$-aminopropionitrile induces degenerative AAs in wild-type mice and azelnidipine prevents aneurysm progression via its anti-inflammatory effect. The anti-inflammatory effect of azelnidipine caused the decreased expression levels of TNF- $\alpha$, MMP-2, and MMP-9, and it conversely enhanced gene expression level of vascular SIRT-1. Vascular SIRT-1 possesses cardiovascular protective properties and improves the function of vascular smooth muscle and vascular endothelial cells [71]. Other experiments have been performed in rat model to investigate the inflammatory basis of TAA. Johnston et al. [72] induced TAA using elastace in mice deficient of IL- $1 \beta$ (IL- $1 \beta$ knockout) or IL- 1 receptor (IL-1R knockout), and they concluded that genetic and pharmacological inhibition of IL- $1 \beta$ decreased TAA formation and progression, indicating that IL- $1 \beta$ may have an important role in TAA formation and complications. Accordingly, decreased IL- $1 \beta$ secretion by thoracic aortic cells in vitro with $\mathrm{z}-\mathrm{VAD}$ suggests a critical upstream role for caspase in TAA 
IL- $1 \beta$ production. Z-VAD is a potent inhibitor of caspase, which cleaves inactive pro-IL-1 $\beta$ to its active form [73]. Caspase activation occurs as part of the inflammasome, a large multiprotein complex containing the NLRP3 protein responsible for caspase activation [74]. The NLRP3 inflammasome is known to play a role in multiple inflammatory conditions and is increased in TAAs [75]. The presence of NLRP3 and caspase in experimental TAA sections, along with the decrease in IL- $1 \beta$ production with caspase inhibition, suggests that caspase and the NLRP3 inflammasome are potential alternative target upstream of IL- $1 \beta$ production that may be useful for further investigation of TAA treatment in order to prevent deadly complications. Finally, another proof that the inflammation has an important role in TAA complications has been drawn by studies regarding mesenchymal stem cells (MSCs) for treatment of aortic aneurysm in rat [76]. The mechanism by which MSCs stabilized aneurysm and prevent dissection is related to their capacity to differentiate, in vitro, in vascular smooth muscle cells (VSMCs) with subsequent reduction of inflammatory reaction. In fact, the most accepted recent pathophysiological hypothesis on TAA proposes the key role of stress- and stretch-induced pathways both in endothelial cells (ECs) lining vasa vasorum and vascular aortic smooth muscle cells (VSMCs) activated by biochemical/mechanical inciting stimuli (i.e., first of all, hypertension, hemodynamic force, smoking and an overload of lipids, etc.) [77]. Their activation created a typical phenotypic switching both in EC and VSMC determining production and release of numerous mitotic and trophic factors, proteoglycans, and metalloproteinases (MMPs), such as MMP-2 and MMP-9 [31]. As result, different cellular and extracellular mechanisms are induced and determine sporadic TAA onset and progression as a multifactorial process with several steps. Among these, inflammatory/immune cellular infiltration in adventitia and media of the aortic wall, oxidative stress, and apoptosis appear principally involved [78]. Persistence of stress or stretch abnormalities results in continued upregulation of these pathways, and ultimately it leads to a proteoglycan accumulation, an increased vascular volume in vasa vasorum, and degradation of elastic fibres with consequent alterations of structure and composition of vascular ECM. Consequent manifestations are the dissection followed or associated with expansion and rupture of the aortic wall.

\section{Conclusions}

Thoracic aortic aneurysm might be advocated as a new emerging inflammatory disease. Evidences suggest that $\mathrm{T}$ lymphocytes and macrophages are the most important inflammatory cells involved in TAA pathogenesis. In addition, a lot of cytokines seem to be involved in the medial degenerative process (loss of smooth muscle cells, elastic fragmentation, and medionecrosis) that induces TAA progression and $\mathrm{AAD}$ onset. In particular, the TGF- $\beta$ signalling pathways have been proposed as an important target involved in both syndromic and sporadic TAA. It is evident that a new therapeutic treatment with statin and anti- inflammatory drug should be proposed in order to prevent aneurysm progression and its lethal complication.

\section{Conflicts of Interest}

All authors declare that they have no competing interests.

\section{Authors' Contributions}

Calogera Pisano and Giovanni Ruvolo take responsibility for all aspects of the reliability and freedom from bias of the data presented and their discussed interpretation.

\section{References}

[1] E. G. Lakatta and S. J. Sollott, "Perspectives on mammalian cardiovascular aging: humans to molecules," Comparative Biochemistry and Physiology Part A: Molecular \& Integrative Physiology, vol. 132, pp. 699-721, 2002.

[2] E. G. Lakatta and D. Levy, "Arterial and cardiac aging: major shareholders in cardiovascular disease enterprises: part II: the aging heart in health: links to heart disease," Circulation, vol. 107, no. 2, pp. 346-354, 2003.

[3] T. Fulop, A. Larbi, J. M. Witkowski et al., "Aging, frailty and age-related diseases," Biogerontology, vol. 11, no. 5, pp. 547563, 2010.

[4] E. Braunwald, "The war against heart failure: the lancet lecture," The Lancet, vol. 385, no. 9970, pp. 812-824, 2015.

[5] Y. A. Chiao and P. S. Rabinovitch, "The aging heart," Cold Spring Harbor Perspectives in Medicine, vol. 7, no. 9, article a025148, 2015.

[6] D. F. Dai, T. Chen, S. C. Johnson, H. Szeto, and P. S. Rabinovitch, "Cardiac aging: from molecular mechanisms to significance in human health and disease," Antioxidants \& Redox Signaling, vol. 16, no. 12, pp. 1492-1536, 2012.

[7] M. Steenman and G. Lande, "Cardiac aging and heart disease in humans," Biophysical Reviews, vol. 9, no. 2, pp. 131-137, 2017.

[8] K. Sharma and D. A. Kass, "Heart failure with preserved ejection fraction: mechanisms, clinical features, and therapies," Circulation Research, vol. 115, no. 1, pp. 79-96, 2014.

[9] K. M. Keller and S. E. Howlett, "Sex differences in the biology and pathology of the aging heart," Canadian Journal of Cardiology, vol. 32, no. 9, pp. 1065-1073, 2016.

[10] J. B. Strait and E. G. Lakatta, "Aging-associated cardiovascular changes and their relationship to heart failure," Heart Failure Clinics, vol. 8, no. 1, pp. 143-164, 2012.

[11] H. A. Feridooni, A. E. Kane, O. Ayaz et al., "The impact of age and frailty on ventricular structure and function in C57BL/6J mice," The Journal of Physiology, vol. 595, no. 12, pp. 37213742, 2017.

[12] A. M. Katz and E. L. Rolett, "Heart failure: when form fails to follow function," European Heart Journal, vol. 37, no. 5, pp. 449-454, 2016.

[13] C. S. Lam, M. Rienstra, W. T. Tay et al., "Atrial fibrillation in heart failure with preserved ejection fraction: association with exercise capacity, left ventricular filling pressures, natriuretic peptides, and left atrial volume," JACC: Heart Failure, vol. 5, no. 2, pp. 92-98, 2017.

[14] H. Yamada, N. Sakata, H. Wada, T. Tashiro, and E. Tayama, "Age-related distensibility and histology of the ascending aorta 
in elderly patients with acute aortic dissection," Journal of Biomechanics, vol. 48, no. 12, pp. 3267-3273, 2015.

[15] M. S. Dzeshka, G. Y. Lip, V. Snezhitskiy, and E. Shantsila, "Cardiac fibrosis in patients with atrial fibrillation: mechanisms and clinical implications," Journal of the American College of Cardiology, vol. 66, no. 8, pp. 943-959, 2015.

[16] C. R. Balistreri, E. Maresi, C. Pisano et al., "Identification of three particular morphological phenotypes in sporadic thoracic aortic aneurysm: phenotype III as sporadic thoracic aortic aneurysm biomarker in aged individuals," Rejuvenation Research, vol. 17, no. 2, pp. 192-196, 2014.

[17] C. Rapezzi, G. Merlini, C. C. Quarta et al., "Systemic cardiac amyloidoses: disease profiles and clinical courses of the 3 main types," Circulation, vol. 120, no. 13, pp. 1203-1212, 2009.

[18] P. van Ooij, J. Garcia, W. V. Potters et al., "Age-related changes in aortic 3D blood flow velocities and wall shear stress: implications for the identification of altered hemodynamics in patients with aortic valve disease," Journal of Magnetic Resonance Imaging, vol. 43, no. 5, pp. 12391249, 2016.

[19] C. R. Balistreri, "Genetic contribution in sporadic thoracic aortic aneurysm? Emerging evidence of genetic variants related to TLR-4-mediated signaling pathway as risk determinants," Vascular Pharmacology, vol. 74, pp. 1-10, 2015.

[20] C. R. Balistreri, C. Pisano, T. D'Amico et al., "The role of inflammation in type A aortic dissection: data of a pilot study," European Journal of Inflammation, vol. 11, pp. 269-278, 2013.

[21] I. El-Hamamsy and M. Yacoub, "Cellular and molecular mechanisms of thoracic aortic aneurysms," Nature Reviews Cardiology, vol. 6, no. 12, pp. 771-786, 2009.

[22] R. He, D. C. Guo, A. L. Estrera et al., "Characterization of the inflammatory and apoptotic cells in the aortas of patients with ascending thoracic aortic aneurysms and dissections," The Journal of Thoracic and Cardiovascular Surgery, vol. 131, no. 3, pp. 671-678.e2, 2006.

[23] G. Ruvolo, C. Pisano, G. Candore et al., "Can the TLR-4mediated signaling pathway be a key inflammatory promoter for sporadic TAA?," Mediators of Inflammation, vol. 2014, Article ID 349476, 14 pages, 2014.

[24] C. Pisano, E. Maresi, C. R. Balistreri et al., "Histological and genetic studies in patients with bicuspid aortic valve and ascending aorta complications," Interactive CardioVascular and Thoracic Surgery, vol. 14, no. 3, pp. 300-306, 2012.

[25] C. Pisano, E. Maresi, D. Merlo et al., "A particular phenotype of ascending aorta aneurysms as precursor of type A aortic dissection," Interactive Cardiovascular and Thoracic Surgery, vol. 15, no. 5, pp. 840-846, 2012.

[26] C. Ihling, T. Szombathy, K. Nampoothiri et al., "Cystic medial degeneration of the aorta is associated with p53 accumulation, Bax upregulation, apoptotic cell death, and cell proliferation," Heart, vol. 82, no. 3, pp. 286-293, 1999.

[27] L. F. Hiratzka, M. A. Creager, E. M. Isselbacher et al., "2010 F/ AHA/AATS/ACR/ASA/SCA/SCAI/SIR/STS/SVM guidelines for the diagnosis and management of patients with thoracic aortic disease representative members, 2014 AHA/ACC guideline for the management of patients with valvular heart disease representative members, Sundt TM 3rd," The Journal of Thoracic and Cardiovascular Surgery, vol. 151, no. 4, pp. 959966, 2016.

[28] C. Pisano, C. Rita Balistreri, O. Fabio Triolo, V. Argano, and G. Ruvolo, "Acute type A aortic dissection: beyond the diameter," The Journal of Heart Valve Disease, vol. 25, no. 6, pp. 764-768, 2016.

[29] C. R. Balistreri, C. Caruso, F. Listì, G. Colonna-Romano, D. Lio, and G. Candore, "LPS-mediated production of pro/anti-inflammatory cytokines and eicosanoids in whole blood samples: biological effects of $+896 \mathrm{~A} / \mathrm{G}$ TLR4 polymorphism in a Sicilian population of healthy subjects," Mechanisms of Ageing and Development, vol. 132, no. 3, pp. 86-92, 2011.

[30] N. Kato, Y. Tatara, M. Ohishi et al., "Angiotensin-converting enzyme single nucleotide polymorphism is a genetic risk factor for cardiovascular disease: a cohort study of hypertensive patients," Hypertension Research, vol. 34, no. 6, pp. 728-734, 2011.

[31] X. Zhang, Y. H. Shen, and S. A. Le Maire, "Thoracic aortic dissection: are matrix metalloproteinases involved?," Vascular, vol. 17, no. 3, pp. 147-157, 2009.

[32] P. S. Silva, R. Lacchini, A. Gomes Vde, and J. E. Tanus-Santos, "Pharmacogenetic implications of the eNOS polymorphisms for cardiovascular action drugs," Arquivos Brasileiros de Cardiologia, vol. 96, no. 2, pp. e27-e34, 2011.

[33] G. Andelfinger, B. Loeys, and H. Dietz, "A decade of discovery in the genetic understanding of thoracic aortic disease," Canadian Journal of Cardiology, vol. 32, no. 1, pp. 13-25, 2016.

[34] L. Scola, F. M. Di Maggio, and L. Vaccarino, "Role of TGF- $\beta$ pathway polymorphisms in sporadic thoracic aortic aneurysm: rs900 TGF- $\beta 2$ is a marker of differential gender susceptibility," Mediators of Inflammation, vol. 2014, Article ID 165758, 8 pages, 2014.

[35] S. Kurtovic, V. Paloschi, L. Folkersen, J. Gottfries, A. FrancoCereceda, and P. Eriksson, "Diverging alternative splicing fingerprints in the transforming growth factor- $\beta$ signaling pathway identified in thoracic aortic aneurysms," Molecular Medicine, vol. 17, no. 7-8, pp. 665-675, 2011.

[36] D. Wu, Y. H. Shen, L. Russell, J. S. Coselli, and S. A. LeMaire, "Molecular mechanisms of thoracic aortic dissection," The Journal of Surgical Research, vol. 184, no. 2, pp. 907-924, 2013.

[37] M. E. Lindsay and H. C. Dietz, "Lessons on the pathogenesis of aneurysm from heritable conditions," Nature, vol. 473, no. 7347, pp. 308-316, 2011.

[38] D. G. Molin, D. R. MC, L. J. Wisse et al., “Altered apoptosis pattern during pharyngeal arch artery remodelling is associated with aortic arch malformations in Tgf $\beta 2$ knock-out mice," Cardiovascular Research, vol. 56, no. 2, pp. 312-322, 2002.

[39] M. E. Lindsay, D. Schepers, N. A. Bolar et al., "Loss-of-function mutations in TGFB2 cause a syndromic presentation of thoracic aortic aneurysm," Nature Genetics, vol. 44, no. 8, pp. 922-927, 2012.

[40] M. Maleszewska, J. R. Moonen, N. Huijkman, B. van de Sluis, G. Krenning, and M. C. Harmsen, "IL- $1 \beta$ and TGF- $\beta 2$ synergistically induce endothelial to mesenchymal transition in an NF $\kappa \mathrm{B}$-dependent manner," Immunobiology, vol. 218, no. 4, pp. 443-454, 2013.

[41] H. Pannu, V. T. Fadulu, J. Chang et al., "Mutations in transforming growth factor- $\beta$ receptor type II cause familial thoracic aortic aneurysms and dissections," Circulation, vol. 112, no. 4, pp. 513-520, 2005.

[42] R. J. Akhurst, "The paradoxical TGF- $\beta$ vasculopathies," Nature Genetics, vol. 44, no. 8, pp. 838-839, 2012.

[43] M. Li, N. Luo, Z. Bai, S. Wang, Y. Shi, and X.'e. Fa, “A canine model of multiple organ dysfunction following acute type-A 
aortic dissection," Surgery Today, vol. 42, no. 9, pp. 876-883, 2012.

[44] X. Ju, T. Ijaz, H. Sun et al., "IL-6 regulates extracellular matrix remodeling associated with aortic dilation in a fibrillin-1 hypomorphic $\mathrm{mgR} / \mathrm{mgR}$ mouse model of severe Marfan syndrome," Journal of the American Heart Association, vol. 3, no. 1, article e000476, 2014.

[45] D. Wen, X.-L. Zhou, J.-J. Li et al., "Plasma concentrations of interleukin-6, C-reactive protein, tumor necrosis factor- $\alpha$ and matrix metalloproteinase-9 in aortic dissection," Clinica Chimica Acta, vol. 413, pp. 198-202, 2012.

[46] S. Kasashima, A. Kawashima, Y. Zen et al., "Upregulated interleukins (IL-6, IL-10, and IL-13) in immunoglobulin G4-related aortic aneurysm patients," Journal of Vascular Surgery, vol. 20, 2017.

[47] A. Gallo, A. Saad, R. Ali, A. Dardik, G. Tellides, and A. Geirsson, "Circulating interferon- $\gamma$-inducible Cys-X-Cys chemokine receptor 3 ligands are elevated in humans with aortic aneurysms and Cys-X-Cys chemokine receptor 3 is necessary for aneurysm formation in mice," The Journal of Thoracic and Cardiovascular Surgery, vol. 143, no. 3, pp. 704-710, 2012.

[48] X. Zhou, G. Xiu, Y. Zhu et al., "Bone marrow mesenchymal stem cells modulated the inflammatory response by regulating the expression of IL-4 and RAGE products in the rats with MODS," Zhonghua Wei Zhong Bing Ji Jiu Yi Xue, vol. 29, no. 4, pp. 294-299, 2017.

[49] S. Makita, A. Ohira, R. Tachieda et al., "Behavior of C-reactive protein levels in medically treated aortic dissection and intramural hematoma," The American Journal of Cardiology, vol. 86, no. 2, pp. 242-244, 2000.

[50] M. Schillinger, H. Domanovits, K. Bayegan et al., "C-reactive protein and mortality in patients with acute aortic disease," Intensive Care Medicine, vol. 28, no. 6, pp. 740-745, 2002.

[51] S. M. Yuan, Y. H. Shi, J. J. Wang, F. Q. Lü, and S. Gao, "Elevated plasma D-dimer and hypersensitive C-reactive protein levels may indicate aortic disorders," Revista Brasileira de Cirurgia Cardiovascular, vol. 26, no. 4, pp. 573-581, 2011.

[52] D. Wen, H. Y. Wu, X. J. Jiang et al., "Role of plasma C-reactive protein and white blood cell count in predicting in-hospital clinical events of acute type A aortic dissection," Chinese Medical Journal, vol. 124, no. 17, pp. 2678-2682, 2011.

[53] K. Sakakura, N. Kubo, J. Ako et al., "Peak C-reactive protein level predicts long-term outcomes in type B acute aortic dissection," Hypertension, vol. 55, no. 2, pp. 422-429, 2010.

[54] P. Ohlmann, O. Morel, B. Radulescu et al., "D-dimer in ruling out acute aortic dissection: sensitivity is not $100 \%$," European Heart Journal, vol. 29, no. 6, pp. 828-829, 2008.

[55] G. Sodeck, H. Domanovits, M. Schillinger et al., "D-dimer in ruling out acute aortic dissection: a systematic review and prospective cohort study," European Heart Journal, vol. 28, no. 24, pp. 3067-3075, 2007.

[56] T. Suzuki, A. Distante, A. Zizza et al., "Diagnosis of acute aortic dissection by D-dimer: the International Registry of Acute Aortic Dissection Substudy on Biomarkers (IRAD-Bio) experience," Circulation, vol. 119, no. 20, pp. 2702-2707, 2009.

[57] D. Paparella, P. G. Malvindi, G. Scrascia et al., "D-dimers are not always elevated in patients with acute aortic dissection," Journal of Cardiovascular Medicine, vol. 10, pp. 212-214, 2010.

[58] P. Ohlmann, A. Faure, O. Morel et al., "Lower circulating Sta-Liatest D-Di levels in patients with aortic intramural hematoma compared with classical aortic dissection," Critical Care Medicine, vol. 37, no. 3, pp. 899-901, 2009.

[59] H. Hazui, M. Nishimoto, M. Hoshiga et al., "Young adult patients with short dissection length and thrombosed false lumen without ulcer-like projections are liable to have falsenegative results of $\mathrm{D}$-dimer testing for acute aortic dissection based on a study of 113 cases," Circulation Journal, vol. 70, no. 12, pp. 1598-1601, 2006.

[60] H. Hazui, H. Fukumoto, N. Negoro et al., "Simple and useful tests for discriminating between acute aortic dissection of the ascending aorta and acute myocardial infarction in the emergency setting," Circulation Journal, vol. 69, no. 6, pp. 677-682, 2005.

[61] K. Nagaoka, K. Sadamatsu, T. Yamawaki et al., "Fibrinogen/ fibrin degradation products in acute aortic dissection," Internal Medicine, vol. 49, no. 18, pp. 1943-1947, 2010.

[62] Y. Jo, T. Anzai, K. Ueno et al., "Re-elevation of D-dimer as a predictor of re-dissection and venous thromboembolism after Stanford type B acute aortic dissection," Heart and Vessels, vol. 25, no. 6, pp. 509-514, 2010.

[63] A. Shimony, K. B. Filion, S. Mottillo, T. Dourian, and M. J. Eisenberg, "Meta-analysis of usefulness of d-dimer to diagnose acute aortic dissection," The American Journal of Cardiology, vol. 107, no. 8, pp. 1227-1234, 2011.

[64] H. Kumar, T. Kawai, and S. Akira, "Toll-like receptors and innate immunity," Biochemical and Biophysical Research Communications, vol. 388, no. 4, pp. 621-625, 2009.

[65] C. R. Balistreri, G. Ruvolo, D. Lio, and R. Madonna, "Toll-like receptor-4 signaling pathway in aorta aging and diseases: "its double nature"," Journal of Molecular and Cellular Cardiology, vol. 110, pp. 38-53, 2017.

[66] M. M. Bachschmid, S. Schildknecht, R. Matsui et al., "Vascular aging: chronic oxidative stress and impairment of redox signaling-consequences for vascular homeostasis and disease," Annals of Medicine, vol. 45, no. 1, pp. 17-36, 2013.

[67] M. Wang, R. E. Monticone, and E. G. Lakatta, "Arterial aging: a journey into subclinical arterial disease," Current Opinion in Nephrology and Hypertension, vol. 19, no. 2, pp. 201-207, 2010.

[68] C. R. Balistreri, G. Candore, G. Accardi, G. Colonna-Romano, and D. Lio, "NF- $\kappa$ B pathway activators as potential ageing biomarkers: targets for new therapeutic strategies," Immunity \& Ageing, vol. 10, no. 1, article 24, 2013.

[69] B. C. Tieu, C. Lee, H. Sun et al., "An adventitial IL-6/MCP1 amplification loop accelerates macrophage-mediated vascular inflammation leading to aortic dissection in mice," Journal of Clinical Investigation, vol. 119, no. 12, pp. 3637-3651, 2009.

[70] H. Kurobe, Y. Matsuoka, Y. Hirata et al., "Azelnidipine suppresses the progression of aortic aneurysm in wild mice model through anti-inflammatory effects," The Journal of Thoracic and Cardiovascular Surgery, vol. 146, no. 6, pp. 1501-1508, 2013.

[71] A. Takemura, K. Iijima, H. Ota et al., "Sirtuin 1 retards hyperphosphatemia-induced calcification of vascular smooth muscle cells," Arteriosclerosis, Thrombosis and Vascular Biology, vol. 31, no. 9, pp. 2054-2062, 2011.

[72] W. F. Johnston, M. Salmon, N. H. Pope et al., "Inhibition of interleukin- $1 \beta$ decreases aneurysm formation and progression in a novel model of thoracic aortic aneurysms," Circulation, vol. 9, no. 130, 11 Supplement 1, pp. S51-S59, 2014. 
[73] E. A. Slee, H. Zhu, S. C. Chow, M. MacFarlane, D. W. Nicholson, and G. M. Cohen, "Benzyloxycarbonyl-Val-AlaAsp (OMe) fluoromethylketone (Z-VAD. FMK) inhibits apoptosis by blocking the processing of CPP32," Biochemical Journal, vol. 315, Part 1, pp. 21-24, 1996.

[74] F. Martinon, K. Burns, and J. Tschopp, “The inflammasome: a molecular platform triggering activation of inflammatory caspases and processing of proIL- $\beta$," Molecular Cell, vol. 10, no. 2, pp. 417-426, 2002.

[75] D. Wu, P. Albini, G. Xu et al., "Nlrp3 inflammasome is upregulated in thoracic aortic aneurysms and dissections," Journal of Surgical Research, vol. 172, p. 280, 2012.

[76] A. Yamawaki-Ogata, R. Hashizume, X. M. Fu, A. Usui, and Y. Narita, "Mesenchymal stem cells for treatment of aortic aneurysms," World Journal of Stem Cells, vol. 6, no. 3, pp. 278-287, 2014.

[77] J. A. Elefteriades, "Thoracic aortic aneurysm: reading the enemy's playbook," The Yale Journal of Biology and Medicine, vol. 81, no. 4, pp. 175-186, 2008.

[78] K. Satoh, P. Nigro, and B. C. Berk, "Oxidative stress and vascular smooth muscle cell growth: a mechanistic linkage by cyclophilin A," Antioxidants \& Redox Signaling, vol. 12, no. 5, pp. 675-682, 2010. 


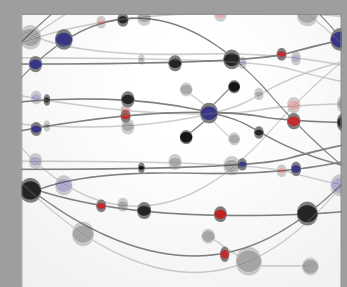

The Scientific World Journal
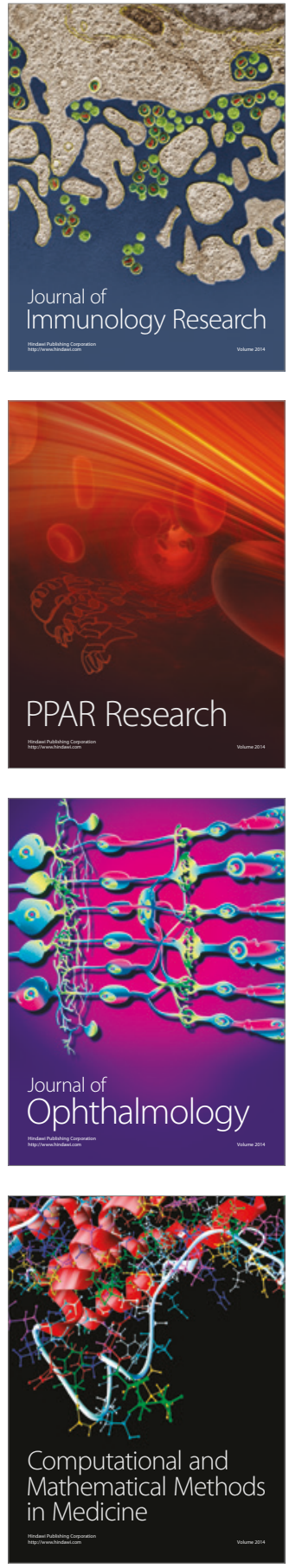

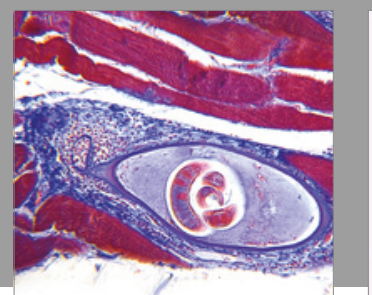

Gastroenterology Research and Practice
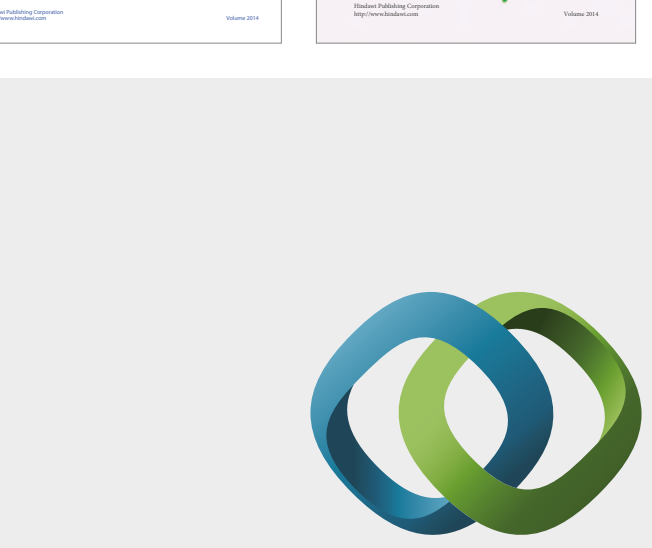

\section{Hindawi}

Submit your manuscripts at

https://www.hindawi.com
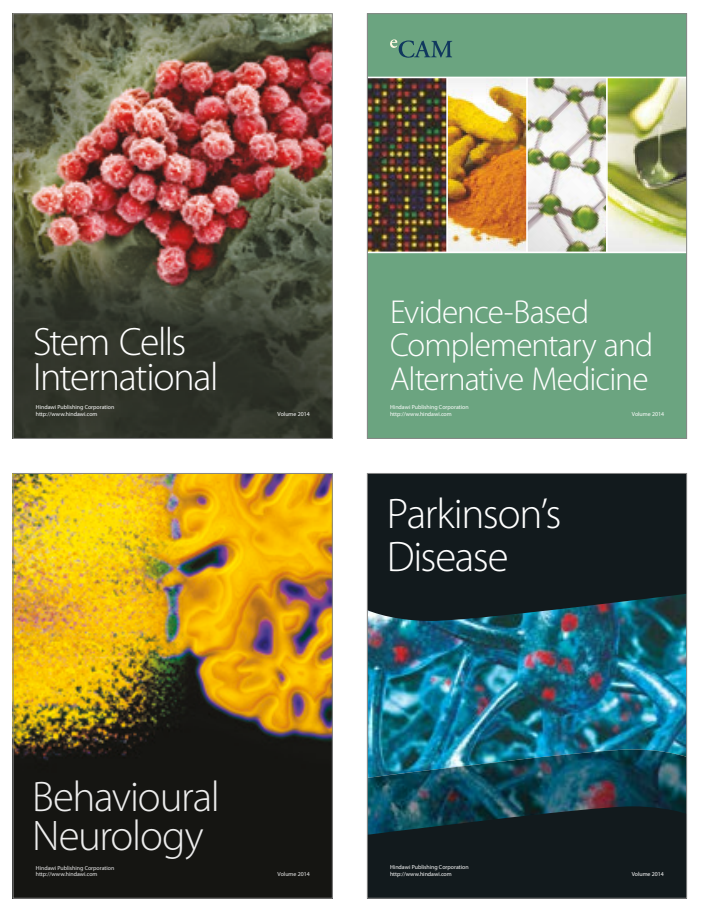
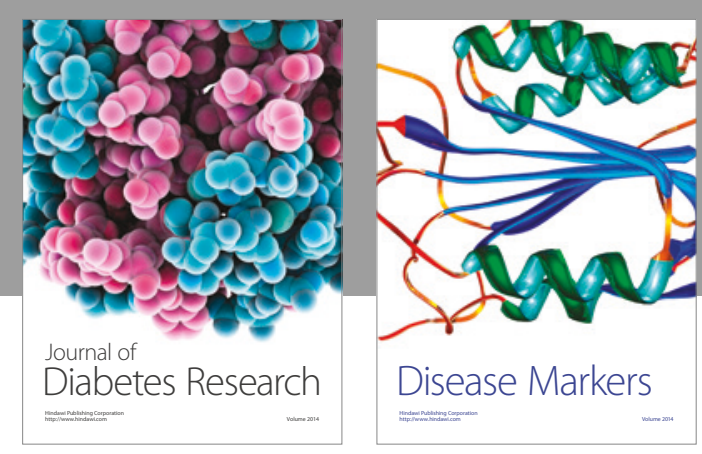

Disease Markers
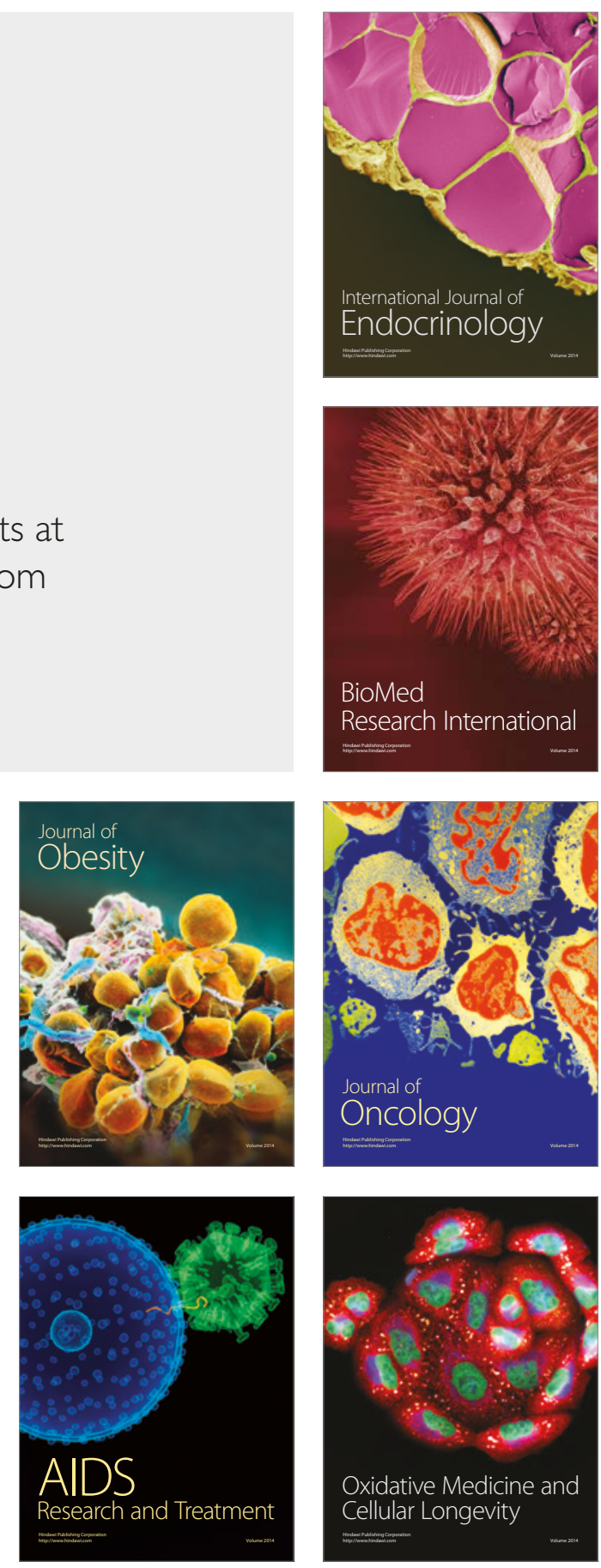